\title{
Hyponatremia with Rhabdomyolysis - Unusual Presentation of Pituitary Apoplexy
}

\author{
Peter George ${ }^{1}$ Neema Montadka Lingappa Gowda ${ }^{2}$
}

\begin{abstract}
:
Hyponatremia is increasingly being identified among hospitalized patients. It significantly alters outcome either by itself or by inappropriate correction. We report a 29-year-old man, who presented to the outpatient services with headache of I week and projectile vomiting of 2 days. He also had cramps in calf since a day. Had no fever and had received intramuscular injection for calf pain. Clinical examination including fundoscopy were normal except for irritability and calf tenderness. Blood chemistry and haematology were normal, except for hyponatremia $(110 \mathrm{mEq} / \mathrm{L}$ ) and elevated CPK (creatine phosphokinase). SIADH was considered as he was euvolemic with low serum osmolality and high urine osmolality. Further evaluation showed hypoadrenalism and hypothyroidism suggestive of hypopituitarism. Subsequent MRI of sella revealed a $1 \mathrm{~cm}^{3}$ cystic mass with features of haemorrhage. Pituitary apoplexy with hypopituitarism was definitive, considering the acute presentation. Supplementation of steroids and thyroxine along with sodium correction improved him symptomatically. Trans-sphenoidal resection of the sellar cystic lesion was performed. Often a good clinical history, assessment and judicious investigations can identify the cause for hyponatremia. Rhabdomyolysis is known to occur in severe hyponatremia and adrenal insufficiency as with the present case. In literature, there are reports of pituitary apoplexy presenting with rhabdomyolysis and hyponatremia. Pituitary apoplexy is a medical emergency which requires early identification, hormonal supplementation and supportive care for better outcome.
\end{abstract}

Key words: Hyponatremia; rhabdomyolysis; pituitary apoplexy; SIADH; hypoadrenalism; hypothyroidism; haemorrhage.

DOI: http://dx.doi.org//0.3329/jom.v20il.38823

Copyright: () 2019 George $P$ et al. This is an open access article published under the Creative Commons Attribution-NonCommercial-NoDerivatives 4.0 International License, which permits use, distribution and reproduction in any medium, provided the original work is properly cited, is not changed in any way and it is not used for commercial purposes.

Received: I/ February, 20I7;

Accepted: 18 May, 2018

\section{Introduction:}

Hyponatremia is frequently identified in clinical practice and known for its variability in clinical presentation. ${ }^{1}$ Rhabdomyolysis associated with hyponatremia though rare, is well described in literature. Hypopituitarism can rarely present with hyponatremia due to underlying hypothyroidism or hypoadrenalism. We report a case of acute hypopituitarism due to pituitary apoplexy presenting as hyponatremia and rhabdomyolysis.

\section{Case Presentation:}

We report a 29-year-old apparently healthy male, who presented to the out-patient services with headache of 1

1. Professor, Department of Medicine, Father Muller Medical College, Mangalore, India.

2. Resident, Department of Medicine, Father Muller Medical College, Mangalore, India.

Corresponding author: Dr. Peter George. Professor, Department of Medicine, Father Muller Medical College, Mangalore, India. Email: drpetergeorge2002@yahoo.com. week and projectile vomiting of 2 days. He also had cramps in calf since a day and had received an intramuscular injection for calf pain. There was no history of fever, seizures or trauma prior to presentation. There was no significant past medical illness. On examination he was irritable and had mild calf tenderness. He was afebrile, well hydrated, with normal muscle tone; he did not have neck stiffness or neurological deficits. The optic fundus appeared normal. Blood chemistry and haematology were normal, except for hyponatremia (110 $\mathrm{m} \mathrm{Eq} / \mathrm{L}$ ) and elevated CPK (4801 IU/L).

As he had euvolemic hyponatremia with low serum osmolality and high urine osmolality, SIADH was considered. For symptomatic hyponatremia he was started on hypertonic saline infusion but had little improvement in sodium levels. Further work up for euvolumic hyponatremia showed hypoadrenalism and hypothyroidism, which was suggestive of hypopituitarism. MR imaging of sella revealed a $1 \mathrm{~cm}^{3}$ cystic mass extending into supra-sellar area indenting the optic chiasma (Figure 1). The features suggestive of 

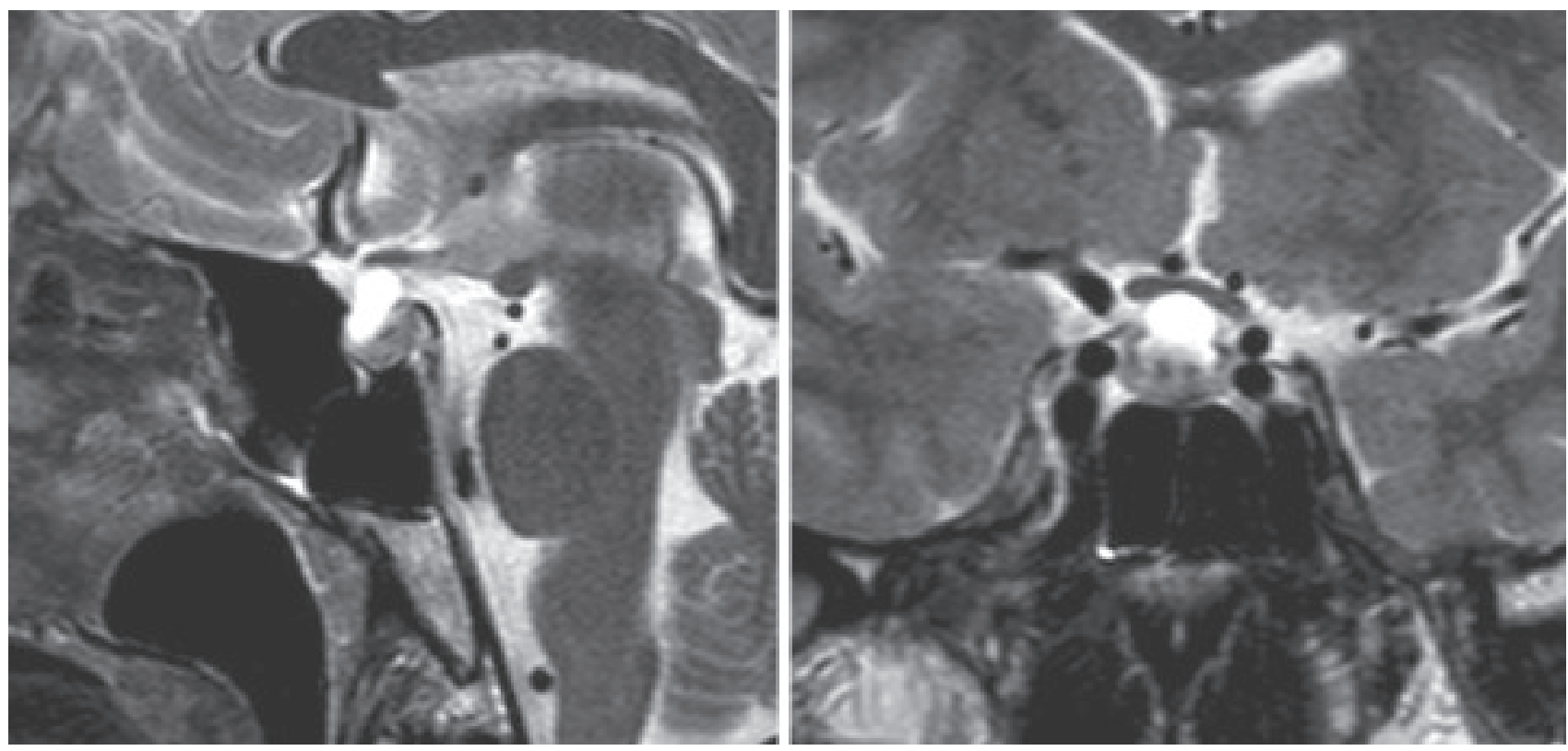

Figure 1: MR sections showing contrast enhanced mass in the sella with cystic changes suggestive of haemorrhage.

haemorrhage to the cystic mass was suggestive of pituitary apoplexy. The acute clinical presentation of hypopituitarism was attributed to pituitary apoplexy. As his prolactin, LH, FSH testosterone levels were normal, pan-hypopituitarism was ruled out.

After establishing the diagnosis, he was started on hormonal replacement with hydrocortisone and thyroxine. He improved symptomatically with sodium and CPK levels returning to near normal levels in 3 days. Trans-sphenoidal resection of the sellar mass done as imaging showed haemorrhage into the lesion and compression to optic chiasma. The resection revealed haemorrhagic fluid in the lesion. He is currently on regular follow up and asymptomatic.

\section{Discussion:}

Hyponatremia is increasingly being identified among hospitalized patients. The symptoms vary from mild upper gastrointestinal symptoms to severe neurological symptoms including seizures, coma and death. ${ }^{1,2}$ Hyponatremia significantly alters patient outcome either by itself, its underlying cause or from inadvertent correction. ${ }^{3}$

The extracellular fluid status of the patient is important to identify the cause, as well in the management of hyponatremia. A good clinical history with assessment, serum osmolality and urinary sodium levels can usually determine the cause. Based on extracellular volume status hyponatremia can be classified as hypovolemic, euvolumic or hypervolumic. ${ }^{3}$ In euvolumic hyponatraemia, the total body sodium remains close to or slightly below normal. The present case presented in severe symptomatic euvolumic hyponatremia.

The common causes for euvolumic hyponatremia are SIADH (syndrome of inappropriate antidiuretic hormone secretion), hypothyroidism, hypoadrenalism and hypopituitarism. ${ }^{2,3} \mathrm{We}$ considered SIADH in the present case as he was euvolemic with low serum osmolality and high urine osmolality. SIADH is considered in patients having euvolemic hyponatremia with low serum osmolality and high urine osmolality, after excluding other causes. Further investigations showed hypothyroidism and hypoadrenalism. Presence of hypothyroidism and hypoadrenalism was suggestive of hypopituitarism and MR imaging identified a cystic mass in pituitary with internal haemorrhage.

Presentation of hyponatremia with rhabdomyolysis is rare. Rhabdomyolysis usually occur following trauma or exposure to toxins. ${ }^{4}$ Among the electrolyte imbalances hyponatremia is rare to cause rhabdomyolysis, whereas hypokalemia and hypocalcemia are frequently implicated. Initially we were unable to correlate hyponatremia with elevated CPK and reasoned it to be from the prior intramuscular injection the patient received. Though literature has described rhabdomyolysis to occur in hyponatremia the exact mechanism is unclear. The mechanism of rhabdomyolysis in hyponatremia is either from alteration in $\mathrm{Na}^{+}-\mathrm{Ca}^{2+}$ pump leading to increase in intracellular $\mathrm{Ca}^{2+}$ or from osmotic injury to cell membrane resulting in extrusion of intracellular potassium. ${ }^{1,3}$ 
As the patient had neurological symptoms of hyponatremia he was started on hypertonic saline infusion along with fluid restriction. The lowering of CPK with correction of hyponatremia suggest hyponatremia to have caused rhabdomyolysis. The CPK levels decreased with sodium correction, but low sodium levels persisted till thyroxine and hydrocortisone was supplemented. Adrenal insufficiency is also described in literature to cause rhabdomyolysis by altering the membrane integrity of sarcolemma. ${ }^{4,5}$

Acute presentation of hypopituitarism is usually due to pituitary infarction or haemorrhage. The term 'pituitary apoplexy' was coined by Brougham et al, to describe clinical features of infarction/ haemorrhage into a pituitary tumour. ${ }^{6}$ It is a potentially fatal medical emergency. The usual symptoms are headache, altered levels of consciousness, visual disturbance, fever or neck stiffness. They may have associated hyponatremia, hypotension, hypoglycaemia and hypothermia depending on the extent of pituitary insufficiency. The usual cause is haemorrhage into a pituitary adenoma as seen with this case. Surgical resection of tumour by trans-sphenoidal route is the definitive treatment. ${ }^{7,8}$ It is performed after correcting the metabolic parameters and adequate hormonal supplementation. Indications for surgery for pituitary adenomas include reduced visual acuity, altered visual field defects, or deteriorating level of consciousness. ${ }^{7,8}$

After correcting the hyponatremia and supplementing with thyroxine and hydrocortisone the patient was symptomatically and clinically better. Trans-sphenoidal resection of the sellar mass revealed haemorrhage into the lesion. He is asymptomatic with hormonal supplementation and is on regular follow up.

Rhabdomyolysis is known to occur in severe hyponatremia and adrenal insufficiency as with the present case. In literature, there are reports of pituitary apoplexy presenting with rhabdomyolysis and hyponatremia. Pituitary apoplexy is a medical emergency, a suspicious clinician, with methodical clinical examination and relevant investigation can easily identify this condition.

\section{Conclusion:}

Rhabdomyolysis due to hyponatremia or adrenal insufficiency are rare to occur independently or together. It is surprising that both these conditions occur in hypopituitarism. We emphasise the importance of a good systematic clinical assessment and investigations in identification of most diseases or disorders encountered in practice.

\section{Conflict of interest: None.}

\section{References:}

1. Verbalis, J.G. Hyponatraemia. Ballieres Clin Endrocrinol Metab 1989;3(2):499-530. https://doi.org/10.1016/S0950351X(89)80013-8

2. Schrier RW \& Berl T. The patient with hyponatremia or hypernatremia. In: Schrier W (ed). Manual of Nephrology, pp 22-37. Philadelphia, Pennsylvania: Lippincott, Williams \& Wilkins; 2009.

3. Sahay M, Sahay R. Hyponatremia: A practical approach. Indian Journal of Endocrinology and Metabolism 2014;18(6):760. https://doi.org/10.4103/2230-8210.141320

4. Vanholder R, Sever MS, Erek E, Lameire N. Rhabdomyolysis. Journal of the American Society of Nephrology 2000;11(8):1553.

5. Adler S. Hyponatremia and rhabdomyolysis: a possible relationship. South Med J 1980;73:511-3. https://doi.org/ 10.1097/00007611-198004000-00029

6. Sasaki M, Yuzawa M, Saito T, Ikoma A, Tamemoto H, Kawakami M, Ishikawa. Clinical and laboratory features of hyponatremia-induced myopathy. Clin Exp Nephrol 2007;11:283-6. https://doi.org/10.1007/s10157-007-0494-3

7. Brougham M, Heusner AP, Adams RD. Acute degenerative changes in adenomas of the pituitary body- with special reference to pituitary apoplexy. J Neurosurg 1950;7:421. https://doi.org/10.3171/jns.1950.7.5.0421

8. Rajasekaran S, Vanderpump M, Baldeweg S, Drake W, Reddy $\mathrm{N}$, Lanyon $\mathrm{M}$, et al. UK guidelines for the management of pituitary apoplexy. Clin Endocrinol (Oxf) 2011;74:9-20. https://doi.org/10.1111/j.1365-2265.2010.03913.x 